\title{
Effects of dexmedetomidine as an adjuvant in thoracic paravertebral block on EC50 of propofol for successful laryngeal mask insertion: a randomized controlled trial
}

\author{
Fei Guo $^{1 \#}$, Hao Chen ${ }^{2 \#}$, Xuejiao Cai ${ }^{1 \#}, J_{i a n l i n} \mathrm{Ge}^{1 \#}$, Boxiang Du ${ }^{1}$, Jie Song ${ }^{1}$ \\ ${ }^{1}$ Department of Anesthesiology, the Second Affiliated Hospital of Nantong University, Nantong, China; ${ }^{2}$ Department of Ultrasound, the Second \\ Affiliated Hospital of Nantong University, Nantong, China \\ Contributions: (I) Conception and design: B Du; (II) Administrative support: H Chen; (III) Provision of study materials or patients: X Cai; (IV) \\ Collection and assembly of data: F Guo; (V) Data analysis and interpretation: J Ge, J Song; (VI) Manuscript writing: All authors; (VII) Final approval \\ of manuscript: All authors. \\ \#These authors contributed equally to this work. \\ Correspondence to: Boxiang Du; Jie Song. Department of Anesthesiology, the Second Affiliated Hospital of Nantong University, Nantong, China. \\ Email: boxiang_du@163.com; jiesong13579@sohu.com.
}

Background: Dexmedetomidine as an adjuvant can improve the duration and the quality of thoracic paravertebral block (TPVB); however, its quantitative effect on propofol infusion is unclear. This study aimed to investigate the effect of dexmedetomidine as an adjuvant in TPVB on the medium effective concentration (EC50) of propofol for successful laryngeal mask insertion.

Methods: Sixty breast cancer patients who underwent elective modified radical mastectomy were enrolled and randomized at a 1:1 ratio into control group (Group C, n=30) or dexmedetomidine group (Group D, $\mathrm{n}=30$ ). Ultrasound-guided T3 paravertebral block was performed before induction of anesthesia. In Group C, $0.5 \%$ ropivacaine $0.3 \mathrm{~mL} / \mathrm{kg}$ was injected into T3 paravertebral space, while subjects in Group D received $0.5 \%$ ropivacaine $0.3 \mathrm{~mL} / \mathrm{kg}$ with dexmedetomidine $(1 \mu \mathrm{g} / \mathrm{kg})$. Propofol target-controlled infusion (TCI) was performed, with an initial target effect-site concentration of $5 \mu \mathrm{g} / \mathrm{mL}$ determined for both groups. The laryngeal mask was inserted once the effect chamber achieved the target concentration. Subsequent target concentrations were adjusted by Dixon up-down sequential method, where dose modifications were performed by $0.5 \mathrm{mg} / \mathrm{mL}$ intervals, based on the success of the laryngeal mask insertion. Probit analysis was used to determine the propofol EC50. Mean arterial pressure (MAP), heart rate (HR), bispectral index (BIS) and application of atropine or ephedrine was recorded. Participants, TPVB giver, and data recorder were blinded to group assignment.

Results: Propofol EC50 for successful laryngeal mask insertion were statistically significant, with $5.256 \mu \mathrm{g} / \mathrm{mL}$ (95\% CI: 4.833, $5.738 \mu \mathrm{g} / \mathrm{mL}$ ) in Group C and $3.172 \mu \mathrm{g} / \mathrm{mL}$ (95\% CI: 2.701, $3.621 \mu \mathrm{g} / \mathrm{mL})$ in Group D. Both groups displayed significantly lower MAP and HR, post propofol TCI $(\mathrm{P}<0.05)$. However, subjects in Group D exhibited lower MAP and HR levels compared to patients in Group $\mathrm{C}(\mathrm{P}<0.05)$. Application of atropine ( $0 \%$ vs. $10 \%)$ and ephedrine (20.0\% vs. $13.3 \%)$ were not significantly different between two groups. Conclusions: Dexmedetomidine, administered as an adjuvant in TPVB, can reduce the TCI concentration of propofol for successful laryngeal mask placement in females. The target concentration of propofol requires adjustment and close monitoring of hemodynamic changes, post induction is warranted.

Trial registration: Chinese Clinical Trial Registry ChiCTR1800016614.

Keywords: Dexmedetomidine; thoracic paravertebral block (TPVB); laryngeal mask; propofol; target-controlled infusion (TCI)

Submitted Jun 16, 2020. Accepted for publication Oct 22, 2020.

doi: $10.21037 /$ atm-20-5174

View this article at: http://dx.doi.org/10.21037/atm-20-5174 


\section{Introduction}

Thoracic paravertebral block (TPVB) refers to the injection of local anesthetic into the paravertebral space to block the corresponding segment of the spinal and sympathetic nerves. It can significantly improve postoperative pain from breast cancer surgery and prevents hemodynamic interference with its unilateral blocking feature. TPVB has attracted much attention in the area of breast cancer analgesia, due to its minimal side effects and potential to facilitate rapid rehabilitation after surgery (1). Currently, clinical studies have shown that thoracic paravertebral block (TPVB) may reduce postoperative opioid consumption (2). With the development and application of ultrasound technology, TPVB technology is also more and more widely used in clinical anesthesia. The mechanism of action of TPVB to relive pain is similar to epidural analgesia which is considered the gold standard for analgesia (3).

Dexmedetomidine is a highly selective $\alpha 2$ adrenergic receptor agonist and exhibits sedative and analgesic effects when used as an auxiliary drug for general anesthesia. Several researches have shown dexmedetomidine as an adjuvant can improve the duration and the quality of never block $(4,5)$. It's has been found that dexmedetomidine can prolong the analgesic duration of local anesthetics when coadministered in TPVB and also reduce the dose of postoperative pain relievers (6).

Targeted-controlled infusion (TCI) is a method of intravenous administration which based on the principles of pharmacokinetics and pharmacodynamics. It can maintain the proper depth of anesthesia by adjusting the drug concentration at the target site (plasma or effect chamber). Intravenous application of dexmedetomidine before induction of anesthesia can reduce the medium effective concentration (EC50) of propofol administration by TCI (7). However, the quantitative effect of dexmedetomidine as a local anesthetic adjuvant in TPVB on propofol by TCI has not been reported. In this study, a randomized controlled trial was conducted to quantify the effect of locally used dexmedetomidine on the EC50 value of propofol in TPVB during successful insertion of the laryngeal mask.

We present the following article in accordance with the CONSORT reporting checklist (available at http://dx.doi. org/10.21037/atm-20-5174).

\section{Methods}

\section{Study design}

This prospective, parallel group, randomized, controlled, double-blind study was approved by the Ethics Committee of the Second Affiliated Hospital of Nantong University (the First People's Hospital of Nantong) (No.004, 2018) and registered on the Chinese Clinical Trial Registry website (ChiCTR1800016614). Written informed consent was provided by each participant or legal guardian. All procedures performed in this study involving human participants were in accordance with the Declaration of Helsinki (as revised in 2013).

A total of 60 patients undergoing major breast cancer surgery, i.e., modified radical mastectomy under general anesthesia at First People's Hospital of Nantong were enrolled and were 1:1 randomly divided into the control group (Group C, n=30) and the dexmedetomidine group (Group D, n=30) by a computer-generated random number table with a block size of four and sealed opaque envelope technique was performed for allocation concealment. The inclusion criteria were comprised of: Aged 18 to 65 years old; ASA class I-II; Mallampati class I-II; $18 \leq \mathrm{BMI} \leq 28 \mathrm{~kg} / \mathrm{m}^{2}$. The exclusion criteria included: Bilateral radical treatment of breast cancer; history of allergy to local anesthetic or propofol; poor control of hypertension, bradycardia, heart block, cerebrovascular disease, diabetes, peripheral nerve disease, mental illness, abnormal liver and kidney function; coagulation disorders, puncture site infection, long-term use of narcotic sedatives or alcohol; gastroesophageal reflux disease and limited cervical range of motion.

\section{Interventions}

\section{Monitoring}

A peripheral venous channel was established for the continuous administration of sodium lactate ringer solution $(6 \mathrm{~mL} / \mathrm{kg} / \mathrm{h})$. ECG, HR, NBP, $\mathrm{SpO}_{2}$ and BIS were monitored throughout the anesthesia and operation.

\section{TPVB}

Ultrasound-guided TPVB was performed at the third thoracic vertebra on the operative side and by a single researcher who was blinded to the group assignment and study drug. After lateral decubitus positioning with the 
operation side up, the in-plane technique was applied through the lateral intercostal approach. The ultrasonic probe was used to identify the intercostal and pleural structures on the para-sagittal section, approximately $3 \mathrm{~cm}$ from the midline. The probe was rotated to produce a long axis parallel to the $3 \mathrm{rd}$ intercostal segment. If necessary, position of the ultrasound probe were adjusted to reveal the transverse processes of the thoracic vertebra at the corresponding segment to identify the pleural structure. A needle to administer the local anesthetic was inserted between the superficial pleura and the intercostal muscle, as guided by the ultrasonic probe. Pleural compression verified the correct position of the injection. Group C was administered a total of $0.5 \%$ ropivacaine (No. LBDX, AstraZeneca AB, Sweden) $0.3 \mathrm{~mL} / \mathrm{kg}$, whilst Group D received $0.3 \mathrm{~mL} / \mathrm{kg}$ of $0.5 \%$ ropivacaine with $1 \mu \mathrm{g} / \mathrm{kg}$ of dexmedetomidine (No. 20170331, Jiangsu Hengrui pharmaceutical Co., Ltd., China).

\section{Propofol TCI induction}

Propofol (No. 1704038, Fresenius Kabi, Germany) administration by TCI pump (Agilia TIVA injection pump, Fresenius Kabi, Germany) was induced 20 minutes after TPVB. The attending anaesthetist was blinded to the patient grouping. The target concentration was set using the modified Dixon up-down sequential method. Based on preliminary results, the initial target concentration of the first patient was set at $5 \mu \mathrm{g} / \mathrm{mL}$. The concentration was modified, based on the success of mask implantation. The target concentration was increased by $0.5 \mu \mathrm{g} / \mathrm{mL}$ and administered to the subsequent patient if the implantation was unsuccessful. Conversely, the target propofol concentration was decreased by $0.5 \mu \mathrm{g} / \mathrm{mL}$ and applied to the subsequent patient upon successful implantation.

\section{Laryngeal mask insertion}

The appropriate laryngeal mask was identified, according to the patient's weight (Igel, Intersurgical, UK). Sizes 3, 4 and 5 were applied to patients within the 30-50, 50-70, and over $70 \mathrm{~kg}$ bodyweight range, respectively. The laryngeal mask was pre-lubricated with lidocaine slurry before insertion. Once the effect-site target concentration was achieved, the laryngeal mask was inserted whilst monitoring the patient's reaction. Implantation was deemed as unsuccessful if the following situations were observed: The patient exhibited a response to a pat on the shoulder or call before laryngeal mask insertion; the patient resisted opening of the mouth; the patient displayed somatosensory reactions, such as cough and laryngeal spasm, during the implantation of laryngeal mask (3). Successful implantation was viewed as the absence of the abovementioned behaviors in each patient. If the laryngeal mask insertion failed, the patient was immediately administered $0.5 \mu \mathrm{g} / \mathrm{kg}$ sufentanil, and $0.15 \mathrm{mg} / \mathrm{kg}$ cisatracurium, if necessary. The attempt to insert the mask was subsequently repeated. After two failed attempts, the patient underwent tracheal intubation under general anesthesia with intermittent positive-pressure ventilation (IPPV). Propofol, remifentanil and cisatracurium were administered intraoperatively to maintain anesthesia and muscle relaxation. Patients were monitored by the anesthesiologists, where the dosing was modified in accordance to the required depth of analgesia, as part of the patient's management.

\section{Recording indicators}

Data were recorded by an anaesthetist who was blinded to the study protocol. MAP, HR and BIS values were recorded at the following time points: T0 (baseline, pre-anesthesia), T1 (during TPVB), T2 (5 min post TPVB), T3 (10 min post TPVB), T4 (20 min after TPVB), T5 (propofol TCI reaching target concentration, pre-laryngeal mask implantation), T6 (1 min post successful laryngeal mask implantation), and T7 (5 min post successful laryngeal mask implantation). A HR $<45 \mathrm{bpm}$ was defined as bradycardia. Intravenous administration of atropine $(0.5 \mathrm{mg})$ was performed, until HR $>45 \mathrm{bpm}$ was achieved. Ephedrine $(6 \mathrm{mg})$ was intravenously administered when a patient was hypotensive, defined by MAP $<60 \mathrm{mmHg}$. Administration ceased when MAP $>60 \mathrm{mmHg}$ was achieved. All concomitant medications, including atropine and ephedrine were recorded throughout the duration of the study.

\section{Statistical analysis}

Data analysis was performed with the SPSS 20.0 statistical software. Based on our preliminary study, the effective concentration for laryngeal mask insertion is $6.5 \pm 2.5 \mu \mathrm{g} / \mathrm{mL}$ in control group and $4.6 \pm 1.9 \mu \mathrm{g} / \mathrm{mL}$ in dexmedetomidine group. With a power of 0.8 and a type I error rate of $5 \%$, the estimated sample size was 23 . Considering a $20 \%$ possible dropout, at least 28 cases in each group was required. Therefore, 30 cases were finally enrolled in each group for analysis. The EC50 and EC95 of propofol for successful laryngeal mask insertion were determined by a modification of Dixon's up-and-down method. Data 


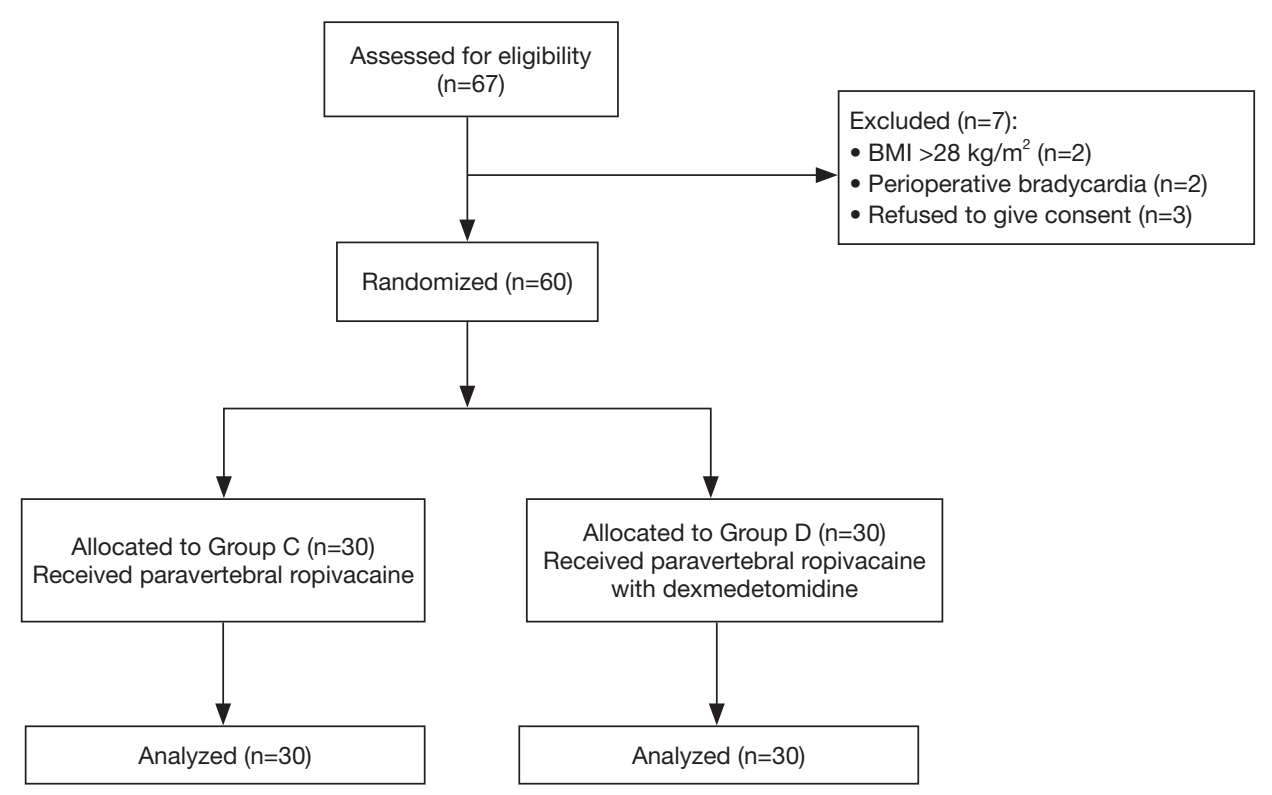

Figure 1 The Consolidated Standards of Reporting Trials (CONSORT) flow diagram of study participants.

were analyzed by using a quantitative response model (Probit analysis) to determine the effective concentration for successful laryngeal mask placement in $50 \%$ and $95 \%$ of patients (EC50 and EC95, respectively). The EC50 difference between groups was evaluated with relative median potency of $95 \%$ CI. Demographic, kidney function, and liver function data were analyzed by independent sample $t$-test. ASA class and Mallampati class comparisons were done using rank sum test. MAP, HR, BIS data were analyzed by repeated measurement analysis of variance. $\mathrm{P}<0.05$ was considered significant.

\section{Results}

\section{General data}

From July 2017 to June 2018, 67 patients were assessed for eligibility, and 7 were excluded. These 7 patients consisted of 2 patients with BMI $>28 \mathrm{~kg} / \mathrm{m}^{2}, 2$ patients had bradycardia and 3 patients who refused to give consent. The remaining 60 patients were randomized in a $1: 1$ ratio: 30 to the control group (Group $\mathrm{C}$ ) and 30 to the dexmedetomidine group (Group D). The Consolidated Standards of Reporting Trials (CONSORT) diagram is shown in Figure 1. Differences between the two groups in general information including age, height, weight, liver and kidney function, ASA and Mallampati class were not statistically significant $(\mathrm{P}>0.05$, Table 1).

\section{Propofol EC50}

Probit analysis was used to determine the EC50 with 95\% CI of propofol by TCI for successful laryngeal mask implantation in both groups. The effect-site EC50 of Group $\mathrm{C}$ was $5.256 \mu \mathrm{g} / \mathrm{mL}$ (95\% CI: 4.833, $5.738 \mu \mathrm{g} / \mathrm{mL}$ ), and the EC95 was $6.512 \mu \mathrm{g} / \mathrm{mL}$ (95\% CI: $5.947,8.310 \mu \mathrm{g} / \mathrm{mL}$ ) (Figure 1). The effect-site EC50 value of Group D was $3.172 \mu \mathrm{g} / \mathrm{mL}(2.701,3.621 \mu \mathrm{g} / \mathrm{mL})$, and the EC95 was $4.428 \mu \mathrm{g} / \mathrm{mL}$ (95\% CI: 3.885, $6.123 \mu \mathrm{g} / \mathrm{mL}$ ) (Figure 2). The relative median potency difference was $2.084 \mu \mathrm{g} / \mathrm{mL}(95 \%$ CI: $0.551,7.430 \mu \mathrm{g} / \mathrm{mL})$. The results of $95 \%$ CI did not contain zero, demonstrating statistical significance in the differences between the two groups.

\section{Changes in MAP and HR}

In this study, the statistical cases at each time point were divided, based on the success of laryngeal mask insertion. For cases with failed laryngeal mask implantation, deep anesthesia was administered to successfully establish an artificial airway, which affected its circulation parameters and BIS value. Therefore, the MAP, HR and BIS values at T6-T7 of these cases were not calculated. No significant differences were observed in MAP and HR between both groups at T0 $(\mathrm{P}>0.05)$. MAP and HR were higher at $\mathrm{T} 1$ compared to T0 in Group D. Additionally, this group displayed significantly lower MAP and HR values at T4- 
Table 1 Comparison of the patients' general data in groups $C$ and $D(n=30)$

\begin{tabular}{|c|c|c|c|c|c|c|c|c|c|c|c|}
\hline Group & $\begin{array}{l}\text { Age } \\
\text { (year) }\end{array}$ & $\begin{array}{c}\text { Height } \\
(\mathrm{kg})\end{array}$ & $\begin{array}{c}\text { Weight } \\
(\mathrm{cm})\end{array}$ & \multicolumn{2}{|c|}{ Liver function } & \multicolumn{2}{|c|}{ Kidney function } & \multicolumn{2}{|c|}{ ASA } & \multicolumn{2}{|c|}{ Mallampati } \\
\hline C & $43 \pm 12$ & $160 \pm 4$ & $64 \pm 9$ & $26 \pm 15$ & $24 \pm 11$ & $58 \pm 13$ & $5.3 \pm 1.3$ & 26 & 4 & 20 & 10 \\
\hline D & $44 \pm 12$ & $162 \pm 5$ & $66 \pm 7$ & $27 \pm 18$ & $26 \pm 14$ & $60 \pm 14$ & $5.0 \pm 1.5$ & 20 & 10 & 22 & 8 \\
\hline
\end{tabular}
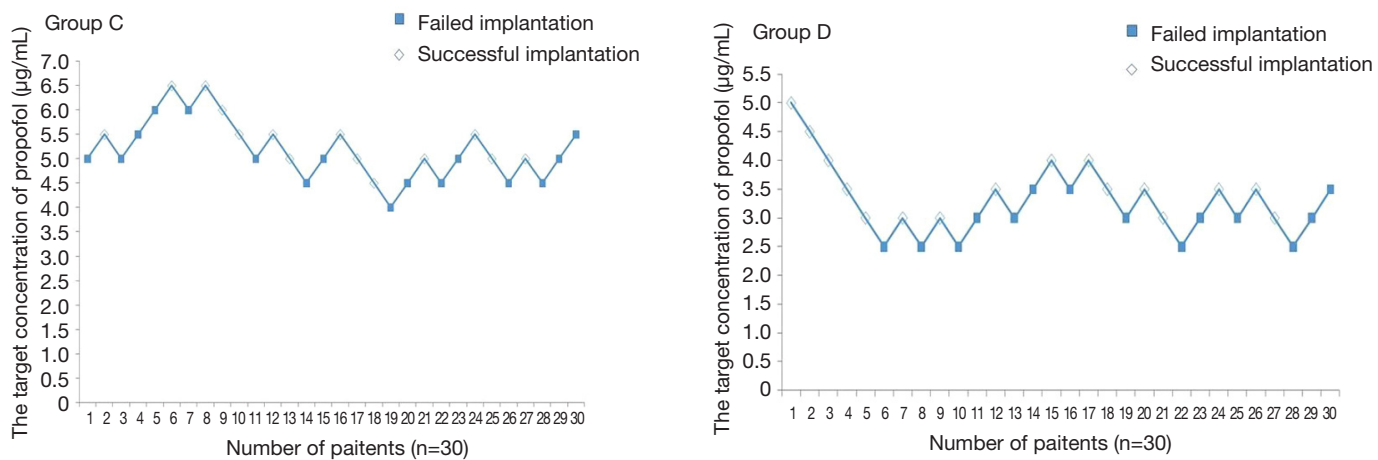

Figure 2 Individual responses to propofol at corresponding effect concentrations. Filled squares represent failed laryngeal mask implantation at the corresponding concentrations. Unfilled diamonds represent failed laryngeal mask implantation at the corresponding concentrations. Group C: received $0.5 \%$ ropivacaine $0.3 \mathrm{~mL} / \mathrm{kg}$; Group D: received $0.3 \mathrm{~mL} / \mathrm{kg}$ of $0.5 \%$ ropivacaine with $1 \mu \mathrm{g} / \mathrm{kg}$ of dexmedetomidine. The EC50 of propofol for successful laryngeal mask insertion was 5.256 and $3.172 \mu \mathrm{g} / \mathrm{mL}$ in the Group C and Group D respectively.

T7, compared to T0 $(\mathrm{P}<0.05)$. There were no statistical differences between MAP and HR at T5 and T6 in Group D (P>0.05) (Table 2). In Group C, MAP and HR were higher than $\mathrm{T} 0$ at $\mathrm{T} 1$, but significantly lower at $\mathrm{T} 5$ compared to $\mathrm{T} 0(\mathrm{P}<0.05)$. A statistical difference between MAP and HR was noted at T5 and T6 $(\mathrm{P}<0.05)$. MAP and HR values at T4-T7 in Group D were significantly lower than that observed in Group C. $(\mathrm{P}<0.05)$ (Table 2). The application of vasoactive drugs was monitored before rather than after the laryngeal mask was inserted. No statistical differences were noted in the application of ephedrine and atropine between the two groups $(\mathrm{P}>0.05)$ (Table 3).

\section{Changes in BIS}

No significant difference in BIS was indicated between the two groups at T0 $(\mathrm{P}>0.05)$. BIS values in Group D were significantly lower at $\mathrm{T} 4$ to $\mathrm{T} 7$, compared to $\mathrm{T} 0(\mathrm{P}<0.05)$. Moreover, the change in BIS between T6 and T5 in the same group was statistically significant $(\mathrm{P}<0.05)$ (Table 2$)$. This phenomenon was also seen in Group $\mathrm{C}$ at the same timepoints $(\mathrm{P}<0.05)$. However, BIS at $\mathrm{T} 5$ and $\mathrm{T} 7$ were significantly lower than T0 $(\mathrm{P}<0.05)$. Timepoints $\mathrm{T} 3$, T4, and T6 are showing lower BIS value in Group D than that in Group $\mathrm{C}(\mathrm{P}<0.05)$ (Table 2).

\section{Discussion}

Patients undergoing breast cancer surgery were selected for inclusion into this trial, where a single point injection in the 3rd thoracic (T3) paravertebral space was selected for TPVB to facilitate potential rapid recovery. In T3 paravertebral space, single point injection can alleviate the postoperative acute pain of breast cancer patients (8), and provides more patient comfort, compared to multipoint injection. Dexmedetomidine has previously shown nil effect on the onset time of TPVB (control group $v s$. dexmedetomidine group: $16.5 \pm 2.6$ vs. $15.3 \pm 2.7 \mathrm{~min}$ ), when locally applied (9). So in our trial, propofol TCI is performed 20 minutes after TPVB to ensure that the TPVB is fully effective. The purpose of this study was to analyze the effect of dexmedetomidine in TPVB on the EC50 of propofol for successful laryngeal mask insertion. Propofol was used without additional medication for mask insertion to minimize the incidence of muscle relaxation or additional opioid effects. The Dixon up-down sequential 
Table 2 Comparison of the patients' MAP, HR and BIS in groups C and D (mean \pm SD)

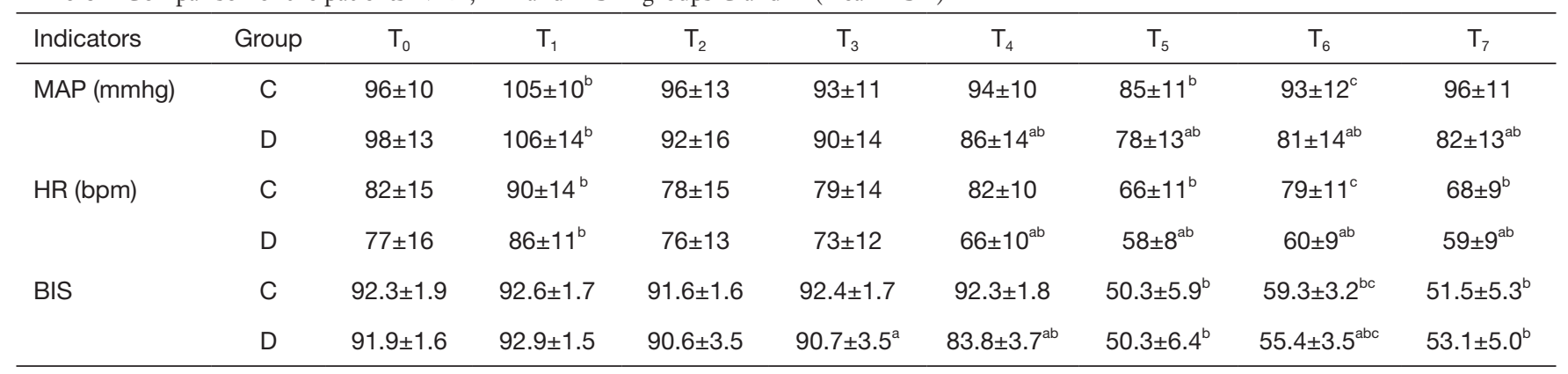

T0-T5, n=30; T6-T7, Group C: $\mathrm{n}=14$, Group D: $\mathrm{n}=16$. ${ }^{\mathrm{a}}, \mathrm{P}<0.05$, compared with Group C; ${ }^{\mathrm{b}}, \mathrm{P}<0.05$, compared with T0; ${ }^{\text {, }}, \mathrm{P}<0.05$, compared with T5. MAP, mean arterial pressure; HR, heart rate; BIS, bispectral index.

Table 3 Number of patients requiring vasoactive drugs in groups $C$ and $\mathrm{D}(\mathrm{n}=30)$

\begin{tabular}{lcc}
\hline Group & Ephedrine, $\mathrm{n}(\%)$ & Atropine, $\mathrm{n}(\%)$ \\
\hline C & $6(20.0)$ & $0(0)$ \\
D & $4(13.3)$ & $3(10)$ \\
\hline
\end{tabular}

method is a simple and effective method to study EC50, with the advantage of being able to fully utilize the data from a small sample size. This method also requires at least 6 inflection points with invalid (failed)/effective (successful) results after sample inclusion (10). Both groups of data in this experiment all met the statistical requirements of the sequential method. The results indicated that $1 \mu \mathrm{g} / \mathrm{kg}$ of dexmedetomidine, administered as a local anesthetic adjuvant through the paravertebral space decreased the EC50 of propofol via TCI by $2.084 \mu \mathrm{g} / \mathrm{mL}$, when the laryngeal mask was successfully implanted without muscle relaxant.

Clinical studies have confirmed that dexmedetomidine used as a local anesthetic adjuvant in nerve block can enhance the local anesthetic effect and prolong the analgesic duration. However, the incidences of slow heart rate and hypotension were observed to increase (11), indicating that dexmedetomidine can exert systemic effects through local absorption. Therefore, the effect of local dexmedetomidine on propofol infusion in general anesthesia is a clinical issue worthy of attention. Local intramuscular injection of $1 \mu \mathrm{g} / \mathrm{kg}$ dexmedetomidine can reduce the concentration of propofol by TCI during induction and surgery (12). This was attributed to the fact that intramuscular injection of dexmedetomidine can be quickly absorbed with a bioavailability of up to $73 \% \pm 11 \%$, eliciting a sedative effect $(13,14)$. In this study, the application of dexmedetomidine in the paravertebral space also reduced the EC50 of propofol by TCI and decreased the BIS value. This could also be attributed to the central effect of the dexmedetomidine injection into the paravertebral space through absorption by the body. However, the pharmacokinetics and pharmacodynamics of locally administered dexmedetomidine in the paravertebral region, as well as the differences with intramuscular injection, need to be further studied.

Statistically, no significant differences were observed between the two groups in the basic values of MAP and HR. When compared to T0, these values were higher at T1, which may be related to the local stimulation during nerve block. In Group D, decreases in MAP and HR were initially observed at T4, which was related to the effects of dexmedetomidine i.e., sedation, decreased heart rate and antihypertension. The blood pressure in both groups at T5-T7 were significantly lower than at baseline, attributed to the inhibitory effect of propofol induction. Compared with T5, MAP and HR values of T6 increased in Group $\mathrm{C}$, which was related to the stimulation of laryngeal mask implantation. However, no significant difference was noted between the two time points in Group D, and the BIS value was significantly lower than that of Group C when the laryngeal mask was inserted, indicating that dexmedetomidine elicited an inhibitory effect on the stress response of laryngeal mask implantation. Although a previous study demonstrated that the intramuscular administration of dexmedetomidine could avoid substantial hemodynamic changes (12), the adverse reactions associated with the lowering of heart rates and blood pressure warrants close monitoring when applied locally. In this study, 4 cases of hypotension and 3 cases of slow heart rate in Group D required treatment, compared with 6 cases and 0 cases in 
the control group, respectively. Although dexmedetomidine was used in Group D, the dosage of propofol was relatively low. Therefore, no statistical difference was observed in cases requiring treatment with vasoactive drugs between the two groups. Dexmedetomidine-induced hypotension and bradycardia were treated with ephedrine and atropine after its administration in this study.

This study investigated the effect of local application of dexmedetomidine on the EC50 of propofol by TCI in the successful implantation of the laryngeal mask. However, some limitations have been noted. Firstly, there are many influencing factors of propofol EC50, including gender (15), age (16), intraoperative fluid supplementation (17), and the female hormonal fluctuations associated with the menstrual cycle (18), etc. All breast cancer patients included in this study were female, without any statistical difference in age between both groups. The types and speed of intraoperative fluid supplementation were preset, but the varying factors associated with the female menstrual cycle were not regulated. Secondly, the pharmacokinetics of dexmedetomidine administered into the paravertebral space has not been elucidated, which will be further investigated in future trials. The purpose of this study was to provide a reference for clinical propofol by TCI. Adding dexmedetomidine as an adjuvant to local anesthetics in TPVB can significantly reduce the TCI concentration of propofol when the laryngeal mask is placed. However, the dexmedetomidine dose, general condition of the patient, presence of opioids or muscle relaxants and the type of laryngeal mask will affect the clinical use of dexmedetomidine. Clinical analysis should be carried out according to the specific conditions.

In summary, dexmedetomidine as a local anesthetic adjuvant for TPVB significantly reduced propofol EC50 when the laryngeal mask was successfully implanted. In clinical application, the dosage of propofol should be reduced and the hemodynamic changes should be noted.

\section{Acknowledgments}

Funding: This study was supported by Science and Technology Project of Nantong (YYZ17084) and Scientific Research Project of Nantong Health Commission (MA2020003).

\section{Footnote}

Reporting Checklist: The authors have completed the
CONSORT reporting checklist. Available at http://dx.doi. org/10.21037/atm-20-5174

Data Sharing Statement: Available at http://dx.doi. org/10.21037/atm-20-5174

Conflicts of Interest: All authors have completed the ICMJE uniform disclosure form (available at http://dx.doi. org/10.21037/atm-20-5174). The authors have no conflicts of interest to declare.

Ethical Statement: The authors are accountable for all aspects of the work in ensuring that questions related to the accuracy or integrity of any part of the work are appropriately investigated and resolved. This randomized controlled study was approved by the Ethics Committee of the Second Affiliated Hospital of Nantong University (the First People's Hospital of Nantong) (No. 004, 2018). Written informed consent was provided by each participant or legal guardian. All procedures performed in this study involving human participants were in accordance with the Declaration of Helsinki (as revised in 2013).

Open Access Statement: This is an Open Access article distributed in accordance with the Creative Commons Attribution-NonCommercial-NoDerivs 4.0 International License (CC BY-NC-ND 4.0), which permits the noncommercial replication and distribution of the article with the strict proviso that no changes or edits are made and the original work is properly cited (including links to both the formal publication through the relevant DOI and the license). See: https://creativecommons.org/licenses/by-nc-nd/4.0/.

\section{References}

1. Pei L, Zhou Y, Tan G, et al. Ultrasound-Assisted Thoracic Paravertebral Block Reduces Intraoperative Opioid Requirement and Improves Analgesia after Breast Cancer Surgery: A Randomized, Controlled, Single-Center Trial. PLoS One 2015;10:e0142249.

2. Kaya FN, Turker G, Mogol EB, et al. Thoracic paravertebral block for video-assisted thoracoscopic surgery: single injection versus multiple injections. J Cardiothorac Vasc Anesth 2012;26:90-4.

3. Thavaneswaran P, Rudkin GE, Cooter RD, et al. Brief reports: paravertebral block for anesthesia: a systematic review. Anesth Analg 2010;110:1740-4.

4. Pintaric TS, Potocnik I, Hadzic A, et al. Comparison of 
continuous thoracic epidural with paravertebral block on perioperative analgesia and hemodynamic stability in patients having open lung surgery. Reg Anesth Pain Med 2011;36:256-60.

5. Baidya DK, Khanna P, Maitra S. Analgesic efficacy and safety of thoracic paravertebral and epidural analgesia for thoracic surgery: a systematic review and meta-analysis. Interact Cardiovasc Thorac Surg 2014;18:626-35.

6. Wang K, Wang LJ, Yang TJ, et al. Dexmedetomidine combined with local anesthetics in thoracic paravertebral block: A systematic review and meta-analysis of randomized controlled trials. Medicine 2018;97:e13164.

7. Yoo JY, Kwak HJ, Kim YB, et al. The effect of dexmedetomidine pretreatment on the median effective bolus dose of propofol for facilitating laryngeal mask airway insertion. J Anesth 2017;31:11-7.

8. Kairaluoma PM, Bachmann MS, Korpinen AK, et al. Single-injection paravertebral block before general anesthesia enhances analgesia after breast cancer surgery with and without associated lymph node biopsy. Anesth Analg 2004;99:1837-43.

9. Chen Y, Liu Y, Li F, et al. The application of thoracic paravertebral nerve block with Dexmedetomidine and Ropivacaine in unilateral thoracic operation. Journal of Clinical Anesthesiology 2015;08:783-5.

10. Park HJ, Lee JR, Kim CS, et al. Remifentanil halves the EC50 of propofol for successful insertion of the laryngeal mask airway and laryngeal tube in pediatric patients. Anesth Analg 2007;105:57-61.

11. Vorobeichik L, Brull R, Abdallah FW. Evidence basis

Cite this article as: Guo F, Chen H, Cai X, Ge J, Du B, Song J. Effects of dexmedetomidine as an adjuvant in thoracic paravertebral block on EC50 of propofol for successful laryngeal mask insertion: a randomized controlled trial. Ann Transl Med 2020;8(22):1480. doi: 10.21037/atm-20-5174 for using perineural dexmedetomidine to enhance the quality of brachial plexus nerve blocks: a systematic review and meta-analysis of randomized controlled trials. Brit J Anaesth 2017;118:167-81.

12. Sun Y, Liu CL, Zhang YH, et al. Low-Dose Intramuscular Dexmedetomidine as Premedication: A Randomized Controlled Trial. Med Sci Monit 2014;20:2714-9.

13. Dyck JB, Maze M, Haack C, et al. The pharmacokinetics and hemodynamic effects of intravenous and intramuscular dexmedetomidine hydrochloride in adult human volunteers. Anesthesiology 1993;78:813-20.

14. Mason KP, Lubisch NB, Robinson F, et al. Intramuscular dexmedetomidine sedation for pediatric MRI and CT. AJR Am J Roentgenol 2011;197:720-5.

15. Xiong M, Zheng Z, Hu ZR, et al. Propofol-sparing effect of different concentrations of dexmedetomidine : Comparison of gender differences. Anaesthesist 2019;68:15-21.

16. Yang N, Zuo MZ, Yue $\mathrm{Y}$, et al. Comparison of $\mathrm{C}_{50}$ for Propofol-remifentanil Target-controlled Infusion and Bispectral Index at Loss of Consciousness and Response to Painful Stimulus in Elderly and Young Patients. Chin Med J (Engl) 2015;128:1994-9.

17. Li Y, Shan Y, Lin X. Effect of acute hypervolemic hemodilution of $6 \%$ hydroxyethyl starch $130 / 0.4$ on the EC50 of propofol at two clinical endpoints in patients. Exp Ther Med 2016;11:110-6.

18. Fu F, Chen X, Feng Y, et al. Propofol EC50 for inducing loss of consciousness is lower in the luteal phase of the menstrual cycle. Brit J Anaesth 2014;112:506-13. 